\title{
Elaboration of the Calculation Algorithm for the Load Diagram of a Thermoelectric Generator that Applies the Temperature Dependent Thermoelectric Layer Properties
}

\author{
Golubev R.O., Stolyarov S.P. \\ Sanct-Petersburg State Marine Technical University \\ Sanct-Petersburg, Russian Federation
}

\begin{abstract}
The aim of the work is the development of the algorithm for the working parameters calculation of a marine waste heat thermoelectric generator, where the thermoelectric layer properties are considered as temperature dependent. The marine thermoelectric generator must have an extensive operational profile that includes a number of modes with a different power demand and different magnitudes of the waste heat parameters. The working mode alteration involves the change in the thermoelectric properties. To achieve the goal a mathematical model was offered for a load diagram calculation. The load diagram represents the generator parameters alteration with respect to the load current change. The most important result was the proposed new order of calculation that differs from the present models with offered variables, an approach to constructional thermal resistances application and a specific electrical power implementation. Mean temperature and load factor are taken as variables with the load factor being accepted as a relation between the electrical resistances of a load and a generator. Constructional thermal resistances were combined into two complex parameters - the thermal resistances of a hot and cold sides. The significance of the obtained results is determined by shaping a versatile approach to the generator`s working parameters calculation. The methodology is independent of the scheme of the calculated generator`s thermal resistances, which could be transformed into the complex parameters. The specific power calculation allows defining the performance per a cross-sectional unit area. The optimal selection of variables reduces the computational time.
\end{abstract}

Keywords: load diagram, thermoelectric generator, thermoelectric module, thermoelectric properties, waste heat recovery system.

DOI: https://doi.org/10.52254/1857-0070.2022.1-53.03

UDC: 621.362

\section{Elaborarea unui algoritm pentru calcularea caracteristicii de sarcină a unui generator termoelectric, ținând cont de dependența de temperatură a proprietăților stratului termoelectric \\ Golubev R.O., Stoliarov S.P. \\ Universitatea tehnică maritimă de Stat din Sanct-Petersburg Sanct-Petersburg, Federația Rusă}

Abstract. Scopul acestei lucrări este de a crea un algoritm pentru calcularea caracteristicilor unui generator termoelectric de utilizare maritimă, în care să ia în considerare dependența de temperatură a proprietăților materialelor termoelectrice utilizate. Generatorul termoelectric de utilizare maritimă trebuie să fie adaptat pentru a funcționa în mai multe moduri de operare, care diferă atât în ceea ce privește parametrii de utilizare a resurselor, cât și aprovizionarea cererii de energie electrică. O schimbare a modului de funcționare atrage după sine o schimbare a proprietăților termoelectrice. Scopul propus este atins prin formularea unui model matematic pentru calculul caracteristicii de sarcină a generatorului termoelectric, prin care se subînțelege o modificare parametrilor agregatului în funcție de curentul de sarcină. Odată cu modificarea curentului de sarcină, se modifică temperaturile joncțiunilor ramurilor generatorului, ceea ce duce la modificarea proprietăților termoelectrice proprii ale ramurilor. Rezultatul cel mai important este crearea unei noi secvențe de calcul al caracteristicilor generatorului, care diferă de modelele matematice existente prin variabilele utilizate, prin abordarea considerării rezistențelor termice ale construcției și prin utilizarea unei puteri specifice în locul puterii totale. Ca mărimi necunoscute se admit temperatura medie între joncțiuni și coeficientul de sarcină - raportul dintre rezistențele electrice ale sarcinii și ale generatorului. Rezistențele termice ale structurii sunt combinate în două complexe - rezistența pe partea caldă şi rezistența pe partea rece. Semnificaţia rezultatelor obținute constă în formarea unei abordări universale pentru calcularea caracteristicilor de performanță ale generatorului. Metodica nu depinde de schema de rezistențe termice dintr-un anumit generator, deoarece acestea în orice caz pot fi reduse la doi parametri complecși - rezistențele de pe ambele părți ale stratului termoelectric.

Cuvinte-cheie: caracteristică de sarcină, generator termoelectric, modul termoelectric, proprietăţi termoelectrice, sistem de recuperare adâncă a căldurii. 


\section{Разработка алгоритма расчёта нагрузочной характеристики термоэлектрического генератора, учитывающего зависимости свойств термоэлектрического слоя от температуры Голубев Р.О., Столяров С.П.}

Санкт-Петербургский государственный морской технических университет Санкт-Петербург, Российская Федерация

Аннотация. Целью работы является создание алгоритма расчёта характеристик судового утилизационного термоэлектрического генератора, в котором обеспечивается учёт температурных зависимостей свойств применяемых термоэлектриков. Термоэлектрический генератор морского исполнения должен быть приспособлен к работе на нескольких эксплуатационных режимах, различающихся как параметрами ресурсов утилизации, так и обеспечиваемой потребностью в электроэнергии. Изменение эксплуатационного режима влечёт за собой и изменение термоэлектрических свойств. Поставленная цель достигается формулированием математической модели расчёта нагрузочной характеристики термоэлектрического генератора, под которой понимается изменение совокупности параметров агрегата в зависимости от тока нагрузки. С изменением тока нагрузки изменяются температуры спаев ветвей генератора, что приводит к изменению термоэлектрических свойств самих ветвей. Наиболее важным результатом является создание новой последовательности расчёта характеристик генератора, отличающейся от уже существующих математических моделей используемыми переменными, подходом к учёту термических сопротивлений конструкции и применением удельной мощности вместо полной. В качестве неизвестных величин принимаются средняя температура между спаями и нагрузочный коэффициент - отношение электрических сопротивлений нагрузки и генератора. Термические сопротивления конструкции объединяются в два комплекса сопротивление по горячей и холодной стороне. Значимость полученных результатов состоит в формировании универсального подхода к расчёту эксплуатационных характеристик генератора. Методика не зависит от схемы термических сопротивлений в данном генераторе, так как они в любом случае могут быть сведены к двум комплексным параметрам - сопротивлениям по обе стороны от термоэлектрического слоя. Расчёт удельной мощности позволяет определить производительность единицы площади поперечного сечения термоэлектрического слоя в условиях отсутствия данных о компоновке генератора. Оптимальный подбор переменных позволяет сократить время расчёта. По данной методике были рассчитаны генераторы с термоэлектрическими слоями из наиболее распространённых сегодня полупроводниковых материалов и показано влияние на характеристики генераторов температурных зависимостей термоэлектрических свойств.

Ключевые слова: нагрузочная характеристика, термоэлектрический генератор, термоэлектрический модуль, термоэлектрические свойства, система глубокой утилизации теплоты.

\section{Введение}

Данная работа посвящена вопросу развития термоэлектрических генераторов (ТЭГ). Непосредственным научным интересом авторов является обеспечение внедрения генераторов данного типа на флоте в качестве элемента системы глубокой утилизации теплоты. На современном этапе развития кораблестроительной промышленности существует потребность в новом типе утилизационных генераторов энергии, способном отвечать актуальным требованиям индустрии. Такой генератор должен быть всережимным, компактным, надёжным в эксплуатации, простым в обслуживании и иметь приемлемую энергоэффективность. В существенной степени этим требованиям отвечают именно ТЭГ.

ТЭГ - это новый тип генераторов не только для кораблестроительной промышленности, но и для энергомашиностроения в целом. Работы по их развитию одновременно ведутся в нескольких направлениях. Поскольку ТЭГ это статический преобразователь тепловой энергии в электрическую, то, на первый взгляд, наиболее очевидным направлением развития генераторов кажется совершенствование свойств термоэлектрических материалов: коэффициента Зеебека ( $\alpha, \mathrm{B} / \mathrm{K})$, удельного электрического сопротивления ( $\rho$, Ом·м), коэффициента теплопроводности ( $\lambda$, $\mathrm{BT} / \mathrm{M} \cdot \mathrm{K})$. Оптимизация свойств направлена на повышение термоэлектрической добротности материала: $Z=\frac{\alpha^{2}}{\rho \lambda}$. На практике, в вопросе применения новых материалов индустрия достаточно консервативна. Например, сплавы теллурида висмута, рассматривавшиеся основателем термоэлектрической теории А.Ф. Иоффе в 50-х годах XX века [1], лишь в конце XX века - начале XXI века начали находить широкое применение в гражданской промышленности. Внедрение новых материалов ограничено и актуальными объёмами промышленного производства сырья. Например, при внедрении систем на базе ТЭГ для утилизации тепловых потерь в промышленности только 
одних США годовой спрос на теллур возрастёт на $34,2 \%$ [2]. По этим и другим причинам, в краткосрочной перспективе, задача внедрения ТЭГ должна решаться на базе существующих материалов.

Существенное число работ посвящено решению частных задач конструирования ТЭГ. Например, в работе [3] был испытан ряд термоэлектрических модулей (ТЭМ, простейшие фабричные сборки термопар) и собран генератор на базе наиболее эффективных из них. В работе [4] решена задача обеспечения электроэнергией оборудования с помощью ТЭГ, устанавливаемого на горячих трубопроводах. Теплоподвод к ТЭГ было предложено осуществлять посредством тепловых трубок. В работе [5] представлены итоги проектно-конструкторской работы по оснащению автомобиля-тягача термоэлектрическими генераторами; описаны задачи, решавшиеся в ходе проектирования.

При проектировании любого ТЭГ необходимо решать задачу интенсификации теплообмена. В работе [6] решается задача оптимизации теплопроводности термоэлектрического слоя с учётом термических сопротивлений конструктивных элементов генератора. Исследование влияния на характеристики ТЭГ таких геометрических параметров теплообменных аппаратов как длина и глубина каналов выполнено в работе [7]. В работе [8] вводится ряд безразмерных комплексов, характеризующих работу ТЭГ. В том числе применяются и комплексы, определяющие условия теплообмена: соотношение конвективных сопротивлений с горячей и холодной сторон, отношение конвективного сопротивления и сопротивления теплопроводности термоэлектрика. Показывается зависимость этих комплексов от температуры и влияние комплексов на производительность генератора. Подробный расчёт оребрения каналов горячей и холодной сред выполнен в работе [9].

Оптимальное использование термоэлектрического материала в конструкции ТЭГ неразрывно связано с учётом зависимости свойств самого материала от температуры. В работах $[10,11,12,13,14,15]$ оптимальное использование температурного градиента обеспечивается за счёт формирования термоэлектрического слоя из разнородных материалов, каждый из которых эффективно работает на определённом участке перепада температур. В работе [10] применение в ветвях термоэлек- трического слоя ТЭГ последовательно нескольких различных материалов позволило достичь КПД в $17 \%$ для температурного перепада в 500 К и КПД в 20,9\% для перепада в 700 К. В работе [11] в ходе испытаний генератора с двухслойными ветвями значение КПД превысило 9\% при разности температур теплоносителей порядка 500 К. Расчёты в работе [12] выполнялись с применением численного моделирования и развитого оптимизационного алгоритма, а при определении термоэлектрических свойств материалов использовались температурные зависимости. Расчёт одного ТЭМ по методике, представленной в работе, занял 80 часов машинного времени. Необходимость поиска оптимального соотношения толщин слоёв ветвей отмечается в работе [13]; обнаружено, что для режимов максимальной мощности и максимально КПД эти соотношения различаются. В работе [14] решается задача соединения разнородных термоэлектрических материалов и показывается влияние существующих технологий на получаемые контактные электрические сопротивления. В работе [15] оптимизация использования теплового потока осуществляется посредством последовательного расположения вдоль вектора температурного градиента каскадов ТЭМ, где в ТЭМ каждого каскада применены различные термоэлектрики.

Проектирование транспортных ТЭГ, в т.ч. и судовых, требует учёта переменности рабочих параметров, основные из которых - это реализуемый перепад температур и соотношение электрических сопротивлений нагрузки и генератора (называемое нагрузочным коэффициентом). Авторами работы [16] были получены для конкретных термоэлектриков квадратичные зависимость максимальной электрической мощности от реализуемого перепада температур. В работах $[17,18]$ выполняется оптимизации ТЭМ в условиях изменения температуры теплоносителя при протекании вдоль модуля. Авторы работы [17] отмечают несоответствие оптимальных режимов работы отдельной термопары и ТЭМ в целом. В работе [18] приводится пример расхождения между эмпирическими и расчётным данными, возникающего при игнорировании температурных зависимостей термоэлектрических свойств. В работе [19] отмечается, что применение при численном моделировании ТЭГ допущения о постоянстве термоэлектрических свойств способно на $80 \%$ сократить время рас- 
чёта. В работе [20] подобные зависимости рекомендуется применять только для ТЭГ, использующих высокотемпературные источники теплоты. В работах [21, 22] приводятся зависимости производительности генераторов от электрического сопротивления нагрузки. Результаты в работе [21] были получены расчётным путём с применением численного моделирования и с учётом температурных зависимостей термоэлектрических свойства; в работе [22] - экспериментом на стенде. Форма кривых в работах [21] и [22] совпадает. Задача оптимизации нагрузочного коэффициента решается в работе [23], отмечается что его оптимальные значение для термоэлектрического слоя и всего ТЭГ не совпадают.

Из перечисленных работ следует, что параметры режима эксплуатации существенно влияют на производительность ТЭГ, а отсутствие учёта температурных зависимостей, применяемых термоэлектрических материалов, может приводить к недостоверному прогнозированию рабочих параметров. Задача учёта температурных зависимостей для спектра режимов ТЭГ решалась в работах $[24,25]$. В обеих работах представлены аналитические решения для расчёта режимов эксплуатации. В аналитической модели работы [24] в качестве неизвестных выступают значения температур конструкции и термоэлектриков. Порядок определения термоэлектрических свойств в условиях отсутствия информации о температурном поле в работе не уточняется. Коэффициенты теплоотдачи предполагаются известными на момент начала расчёта. В работе [25] в качестве неизвестных приняты температуры спаев - торцевых поверхностей термоэлектриков с горячей и холодной сторон. Этими температурами задаются и затем итерационно уточняют. В начале расчёта должны быть известными такие параметры ТЭГ как число термопар, длины и площади поперечных сечений термоэлементов, площадь поверхности керамических пластин.

Данная работа посвящена развитию аналитической модели учёта температурных зависимостей термоэлектрических свойств при расчёте режимов работы генератора. Авторам видится целесообразной реализация в математической модели следующих аспектов:

- в качестве неизвестных величин использовать среднюю температуру между спаями и нагрузочный коэффициент, что должно уменьшить число итераций и повысить точность расчёта;
- ввести понятие тепловых сопротивлений конструкции с горячей и холодной сторон. Этими сопротивлениями могут быть учтены конструктивные особенности каждого рассчитываемого ТЭГ, а область применения математической модели будет существенно расширена;

- определять не полную, а удельную электрическую мощность - приходящуюся на единицу площади поперечного сечения термоэлектрика, благодаря чему применение математической модели не будет ограничиваться конкретным ТЭМ или ТЭГ, а результаты расчёта могут быть масштабированы;

- видится целесообразным режимы работы генератора объединить в нагрузочную характеристику - совокупность зависимостей параметров от тока нагрузки. Она позволяет наглядно представлять зависимость производительности генератора от теплопередачи и термоэлектрических эффектов.

Формулирование математической модели позволит рассчитать нагрузочные характеристики для ряда термоэлектриков широко применяемых в промышленности и выявить степень влияния на режимы работы ТЭГ температурных зависимостей свойств этих материалов.

\section{Математическая модель расчёта нагрузочной характеристики ТЭГ, учитывающая температурные зависимости термоэлектрических свойств}

В разделе приведены основные положения математической модели, сформулированной для автоматизированного построения нагрузочной характеристики ТЭГ.

При построение нагрузочной характеристики используется относительный ток нагрузки $(\bar{I})$ - отношение текущего значение электрического тока к току короткого замыкания. Относительный ток нагрузки изменяется в диапазоне от нуля - холостой ход (XX, нагрузка крайне велика), до единицы - короткое замыкание (КЗ, нагрузка крайне мала). ТЭГ, рассматриваемый на нагрузочной характеристике, представляет из себя некоторую элементарную часть действительного генератора, в пределах которой температура горячей и холодной рабочих сред остаются неизменными. Если в оценочном расчёте перейти от действительных температур теплоносителей, изменяющихся при протекании через генератор, к средним температурам, то возможно ре- 
зультаты для элементарного участка экстраполировать на действительный агрегат. На нагрузочной характеристике приводятся следующие основные параметры работы ТЭГ:

- температура горячих и холодных спаев $\left(T_{h}\right.$ и $\left.T_{c}\right)$ - отличается от температуры рабочих сред на величину потерь в элементах конструкции ТЭГ, расположенных между теплоносителями и соответствующими спаями генератора;

- разность температуры спаев $(\Delta T)$ - перепад температуры между теплоносителями, полезно используемый генератором;

- удельная мощность ТЭГ $\left(N_{\text {spec }}\right)$ - мощность, вырабатываемая генератором с единицы площади поверхности термоэлектрического слоя;

- удельный тепловой поток, подводимый к горячим спаям $\left(q_{h}\right)$ - отличается от теплового потока теплопроводности вследствие наличия термоэлектрических эффектов;

- КПД ТЭГ ( ๆ) - отношение удельной мощности к удельному тепловому потоку на горячих спаях;

- коэффициент термопроводности $\left(k_{T}\right)-$ отношение полного теплового потока к тепловому потоку теплопроводности;

- нагрузочный коэффициент $(m)$ - отношение электрических сопротивлений нагрузки и ТЭГ;

- относительная ЭДС ( $\bar{E})$ - отношение ЭДС на режиме к ЭДС холостого хода;

- относительное напряжение $(\bar{U})$ - отношение падения напряжения на нагрузке к ЭДС холостого хода.

Для проведения расчётов по математической модели необходимы следующие исходные данные: применяемые термоэлектрические материалы, средняя температура горячей и холодной среды $\left(\theta_{h}, \theta_{c}\right)$, конструктивные термические сопротивления ТЭГ с горячей и холодной сторон $\left(R_{h}, R_{c}\right)$ и толщина термоэлектрического слоя $(\delta)$. Схема последовательности расчёта приведена на рис. 1. В общем случае данная математическая модель может использоваться как при постоянных, так и при переменных термоэлектрических свойствах. В обоих случаях, для режимов отличных от XX и КЗ, в качестве неизвестных выступают средняя температура $\left(T_{m}\right)$ и нагрузочный коэффициент. Изначально $T_{m}$ прини- мается оптимальной для данных термоэлектрических материалов. Если температурные зависимости термоэлектрических свойств не учитываются, то при итерационном уточнении $T_{m}$ изменение свойств термоэлектриков не происходит. Для построения нагрузочной характеристики и учёта температурных зависимостей свойств $T_{m}$ достаточно определять с погрешностью в пол градуса.

Из рис. 1 следует, что расчёт состоит из трёх частей: рассчитываются предельные режимы (холостого хода и короткого замыкания); затем для некоторого тока нагрузки рассчитывается необходимый долевой режим работы. Совокупность результатов расчётов предельных режимов и ряда долевых режимов позволяет построить нагрузочную характеристику.

Для режима $\mathrm{XX}$, при принятой в первом приближении средней температуре между горячими и холодными спаями $\left(T_{m}\right)$, определяется коэффициент теплопроводности полупроводникового слоя и затем находится его тепловое сопротивление: $R=\delta / \lambda$. Поскольку в режиме XX ТЭГ работает как обычный теплообменный аппарат, далее перепад температуры между спаями рассчитывается по формуле (1).

$$
\Delta T=\frac{R\left(\theta_{h}-\theta_{c}\right)}{R_{h}+R+R_{c}}
$$

Перечисленных операций достаточно для того, чтобы в первом приближении определить температуру спаев по формулам (2).

$$
T_{h}=\theta_{h}-\frac{\Delta T R_{h}}{R}, T_{c}=\theta_{c}+\frac{\Delta T R_{c}}{R}
$$

По полученной температуре спаев рассчитывается и корректируется средняя температура. Когда достигается требуемая точность её определения, могут быть найдены необходимые параметры режима: удельный тепловой поток на горячем спае $\left(q_{h}=\frac{\theta_{h}-T_{h}}{R_{h}}\right)$; ЭДС режима XX ( $\left.E_{i d l}=\alpha \Delta T\right)$. Коэффициент Зеебека для режима определяется по уточнённому значению $T_{m}$. Относительные ЭДС и напряжения остальных режимов определяются отношением абсолютных значений к значению $E_{i d l}$. 


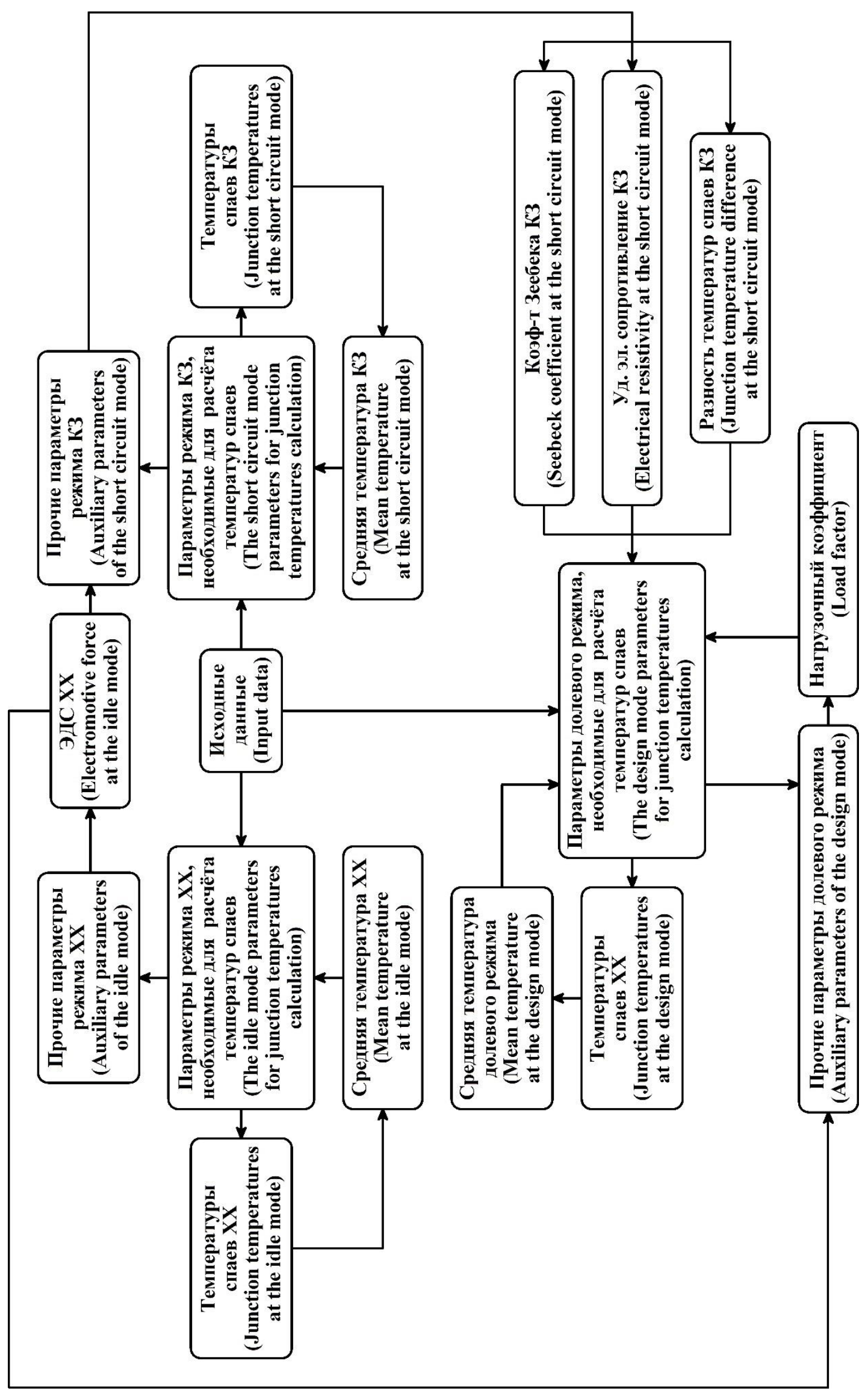

Рис. 1. Схема последовательности расчётов, выполняемых при построении нагрузочной характеристики. ${ }^{1}$ 
Для режима $\mathrm{XX} \bar{E}$ и $\bar{U}$ равны единице. Также единице равен коэффициент термопроводности, т.к. $q_{h}$ равен тепловому потоку теплопроводности. КПД и $N_{\text {spec }}$ имеют нулевые значения.

В отличие от режима $\mathrm{XX}$, в режиме КЗ $q_{h}$ существенно выше теплового потока тепло- проводности, но $\eta$ также равен нулю, поскольку отсутствует сопротивление нагрузки. В этом случае $T_{h}$ может быть найдено из решения квадратного уравнения (3). Для чего предварительно требуется определить термоэлектрические свойства, необходимые для расчёта $Z$. Расчёт также выполняется в несколько последовательных приближений по $T_{m}$.

$$
\begin{aligned}
& \left(R_{h}{ }^{2}-R_{c}{ }^{2}\right) T_{h}{ }^{2}+2\left[\theta_{h} R_{c}{ }^{2}+\theta_{c} R_{h} R_{c}+\frac{R_{h}{ }^{2}+R_{h}\left(R+R_{c}\right)}{Z}\right] T_{h}- \\
& -\left[\theta_{h}{ }^{2} R_{c}{ }^{2}+\frac{2 \theta_{h} R_{h}\left(R+R_{c}\right)}{Z}+2 \theta_{h} \theta_{c} R_{h} R_{c}+\theta_{c}{ }^{2} R_{h}{ }^{2}+\frac{2 \theta_{c} R_{h}{ }^{2}}{Z}\right]=0
\end{aligned}
$$

В отличие от режимов XX и КЗ, для долевых (промежуточных) режимов по току нагрузки нагрузочный коэффициент является неизвестной величиной. В первом приближении его необходимо задать, а далее последовательно определить:

- $\Delta T$ по формуле для относительного тока нагрузки:

$$
\bar{I}=\frac{\rho_{S h C} \alpha \Delta T}{\rho \alpha_{S h C} \Delta T_{S h C}(1+m)},
$$

где величины с индексом « $S h C$ » относятся к ранее рассчитанному режиму короткого замыкания;

• КПД ТЭГ:

$$
\eta=\frac{Z \Delta T^{2} m\left(R_{h}+R_{c}\right)}{(1+m)^{2} R\left(\theta_{h}-\Delta T-\theta_{c}\right)+Z \Delta T^{2} m R_{c}} ;
$$

- при текущем значении $m$ рассчитать удельный тепловой поток $\left(q_{h}\right)$; температуру спаев; уточнить среднюю температуру.

Действительное значение $m$ для ранее рассчитанных параметров может быть затем найдено из квадратного уравнения (6).

$$
m^{2}+\left[2+Z\left(T_{h}-\frac{\Delta T}{\eta}\right)\right] m+Z T_{m}+1=0
$$

Исходное значение $m$ сравнивается с полученным из уравнения (6) и принимается решение о необходимости последующего его уточнения. Например, расчёты показали, что необ- ходимая плавность зависимости $m=f(\bar{I})$ может быть обеспечена при следующих погрешностях: для области значений с $m$ больше единицы допустимая разница значений между результатами, полученными на двух последующих итерациях, не превышает одну десятую; для области от 0,5 до 1 по $m$ - не превышает одну сотую и для области с $m$ менее $0,5-$ не превышает одну пятитысячную.

Когда $m$ и $T_{m}$ получены с удовлетворительной точностью, могут быть рассчитаны остальные параметры для нагрузочной характеристики: удельная мощность по формуле (7), коэффициент термопроводности по формуле (8), относительные ЭДС $\left(\bar{E}=\frac{\alpha \Delta T}{E_{i d l}}\right)$ и напряжение $\left(\bar{U}=\frac{\bar{E} m}{1+m}\right)$ и т.д.

$$
\begin{gathered}
N_{\text {spec }}=\frac{Z \Delta T^{2} \lambda m}{\delta(1+m)^{2}} \\
k_{T}=1+Z \cdot \frac{T_{m}+m T_{h}}{(1+m)^{2}}
\end{gathered}
$$

\section{Температурные зависимости свойств термоэлектриков}

В данном разделе рассматриваются температурные зависимости термоэлектрических свойств ряда материалов, производство которых освоено промышленностью:

- теллурид висмута $\left(\mathrm{Bi}_{2} \mathrm{Te}_{3}\right)$ с р и $\mathrm{n}$ проводимостью (при различном легировании) свойства приняты в соответствии с источником [26]; 
- твёрдые растворы (тройные сплавы): $\mathrm{p}$ ветви изготовлены из $\mathrm{Bi}_{2} \mathrm{Te}_{3}-\mathrm{Sb}_{2} \mathrm{Te}_{3}, \mathrm{n}$ ветви изготовлены из $\mathrm{Bi}_{2} \mathrm{Te}_{3}-\mathrm{Bi}_{2} \mathrm{Se}_{3}$ - свойства приняты в соответствии с источником [27];

- теллурид свинца (PbTe) с р и n проводимостью (при различном легировании) - свойства приняты в соответствии с источником [28].

Применение данных материалов в производстве ТЭГ морского исполнения наиболее вероятно на современном этапе развития отрасли. Материалы имеют различные диапазоны рабочих температур. Для работы ТЭГ на больших температурных перепадах из этих термоэлектриков могут быть произведены многослойные ветви для ТЭМ.

Математической моделью, приведённой выше, предполагается выполнение расчётов для слоя полупроводникового материала. Определение свойств термоэлектрического слоя означает их осреднение между ветвями с электронной (p) и дырочной (n) проводимостью. Оно выполнялось в соответствии с рекомендациями, приведёнными в работах [29, 30]. Для наиболее распространённых П-образных термопар (простейших ТЭГ), подобных приведённой на рис. 2 , высота р- и n-ветвей ( $\delta$ ) одинакова. Некоторый условно однородный слой полупроводникового материала обеспечивается нахождением соотношения площадей поперечных сечений ветвей $\left(A_{p} / A_{n}\right)$, которое

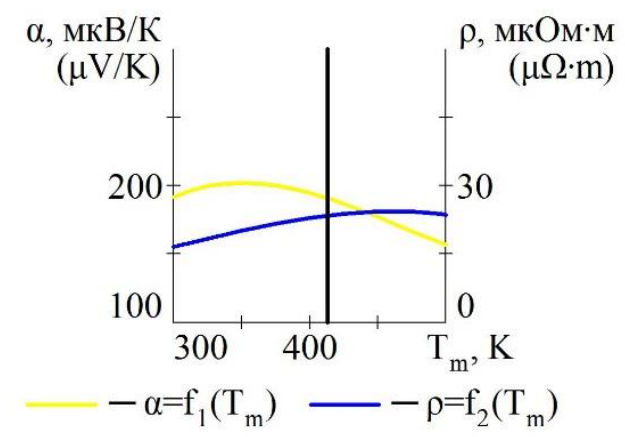

компенсирует разнородность их свойств. В итоге расчёт выполняется для ветви с некоторой условной площадью: $A=\frac{A_{p}+A_{n}}{2}$. Полные мощность и тепловой поток на горячих спаях могут быть найдены домножением удельных значений $\left(N_{s p e c}, q_{h}\right)$ на суммарную площадь поперечных сечений ветвей.

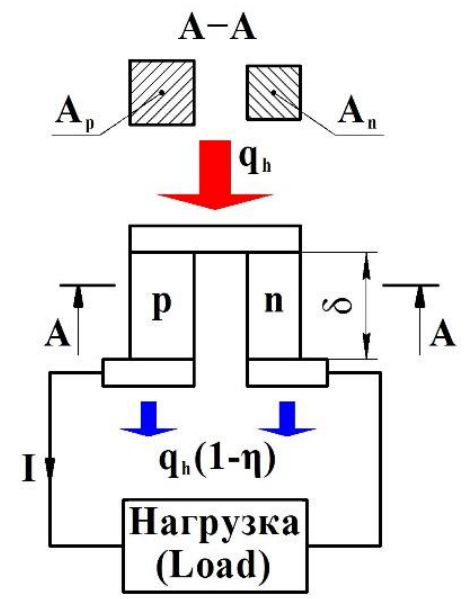

Рис. 2. Принципиальная схема ТЭГ. ${ }^{2}$

На рис. 3 - рис. 5 приведены графические зависимости термоэлектрических свойств полупроводниковых слоёв от средней температуры. Вертикальная чёрная линия на рисунках соответствует расчётной средней эксплуатационной температуре генератора.

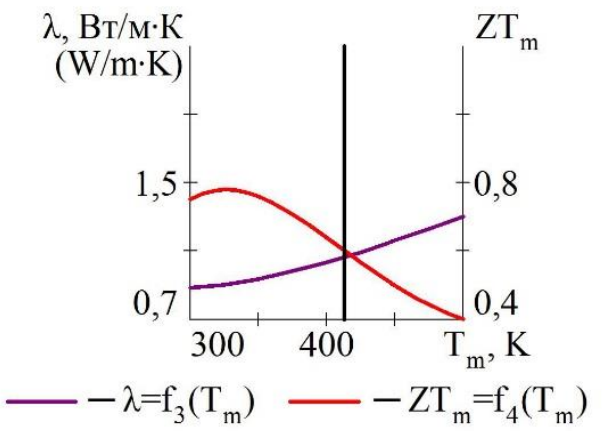

Рис. 3. Термоэлектрические свойства $\mathrm{Bi}_{2} \mathrm{Te}_{3 .}{ }^{3}$
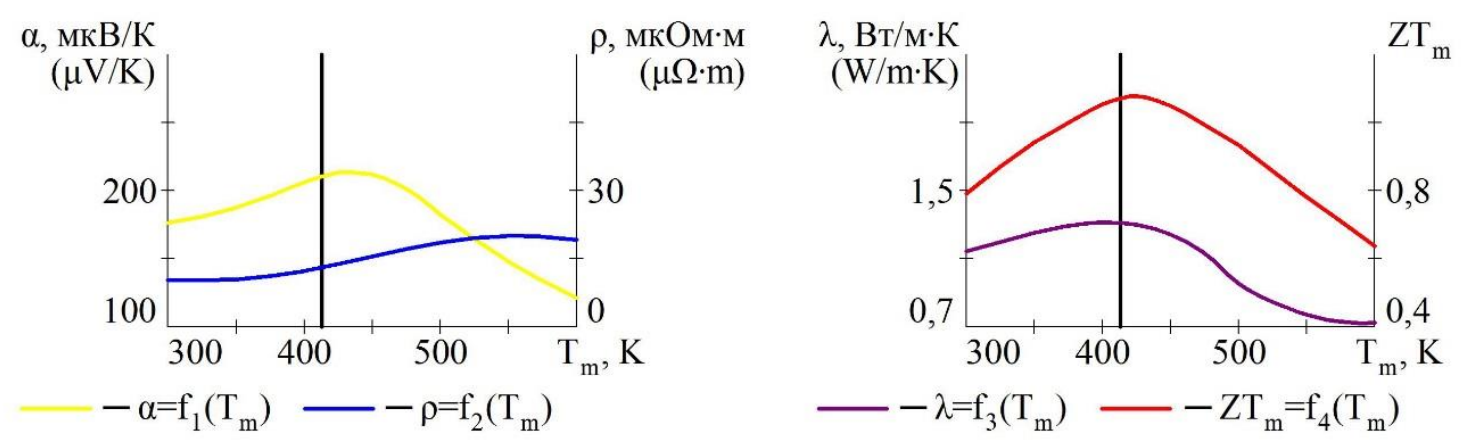

Рис. 4. Термоэлектрические свойства Bi2 $\mathrm{Te}_{3}-\mathrm{Sb}_{2} \mathrm{Te}_{3}$ и $\mathrm{Bi}_{2} \mathrm{Te}_{3}-\mathrm{Bi}_{2} \mathrm{Se}_{3}{ }^{4}$ 

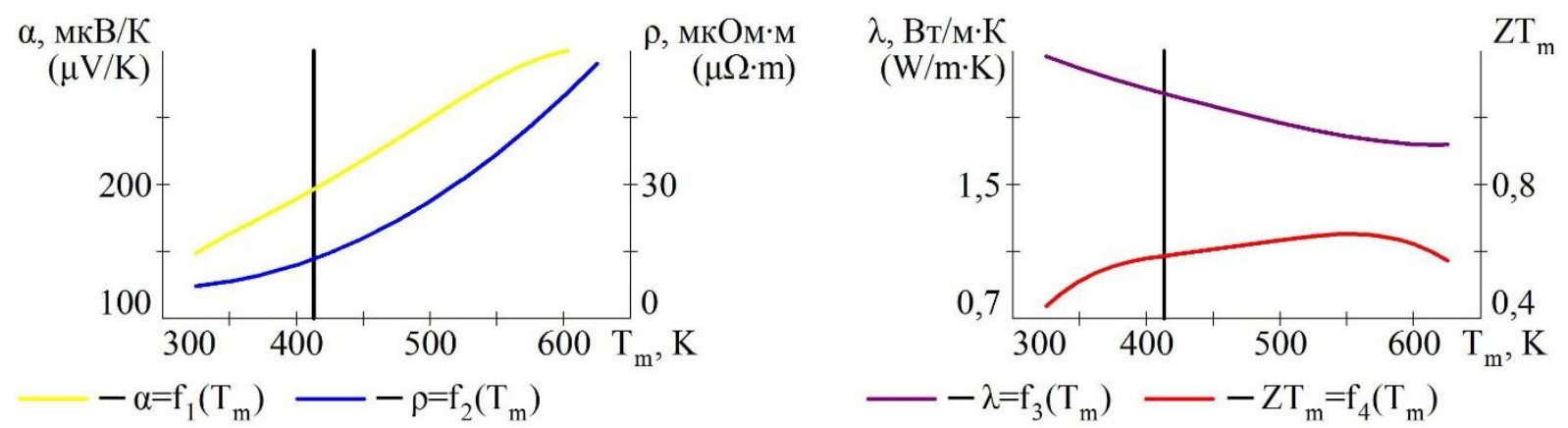

Рис. 5. Термоэлектрические свойства РbТе. ${ }^{5}$

По рис. 3 - рис. 5 видно, что если материал полупроводникового слоя подобран неверно (как на рис. 3 и рис. 5), то термоэлектрическая добротность будет существенно ниже теоретически достижимой. Для слоя на рис. 4 значение добротности на режиме близко к максимальному.

На рис. 6 совмещены безразмерные термоэлектрические добротности рассматриваемых материалов. Из рис. 6 следует, что добротность существенно изменяется от материала к материалу и для одного материала при изменении его температуры.

Важно отметить, что графики на рисунках построены для средней температуры слоя, следовательно, температуры горячих и холодных спаев и тем более самих теплоносителей существенно отличаются от этой температуры как в большую, так и в меньшую сторону. Из графиков следует, что, при определении характеристик ТЭГ в конкретных условиях эксплуатации, необходимо учитывать зависимость термоэлектрических свойств полупроводникового слоя ТЭГ от температуры.

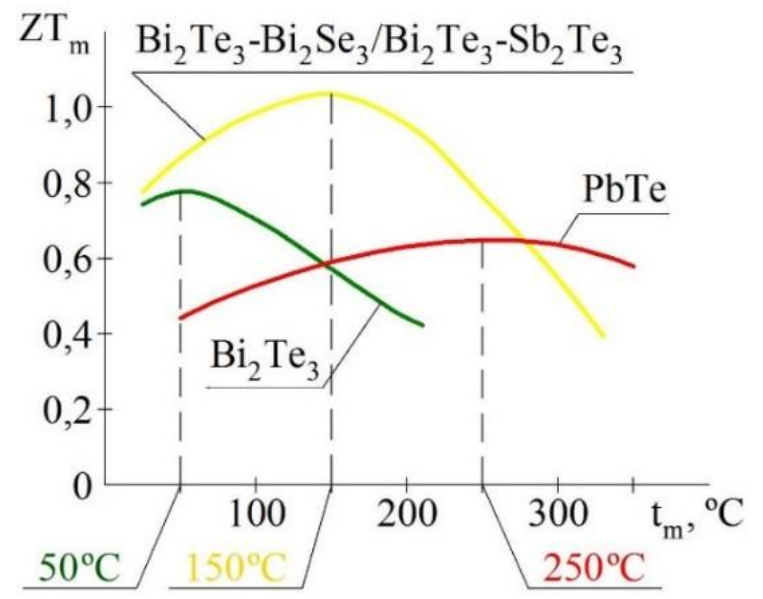

Рис. 6. Безразмерные термоэлектрические добротности. ${ }^{6}$

\section{Нагрузочные характеристики ТЭГ}

Для того чтобы проанализировать влияние температурных зависимостей термоэлектрических свойств на параметры ТЭГ, в которых применяются материалы из предыдущего раздела, были рассчитаны и построены нагрузочные характеристики.

Для каждого генератора расчёт выполнялся в двух вариантах: при переменных и постоянных термоэлектрических свойствах. Во всех случаях характеристики строились для полупроводникового слоя толщиной 2 мм. В расчётах с постоянными термоэлектрическими свойствами их значения принимались по оптимальной средней температуре на рис. 6. Чтобы влияние переменности термоэлектрических свойств на работу ТЭГ было явным, температура горячей и холодной сред принимались таким образом, чтобы действительная средняя температура существенно отличалась от оптимальной. Температура холодной среды во всех случаях принималась равной $30^{\circ} \mathrm{C}$.

Термическое сопротивление конструкции ТЭГ существенно выше, чем отдельной термопары на рис. 2. Если к элементам конструкции отнести все части ТЭГ, кроме термоэлектрического слоя, то, в соответствии с рис. 7, свой вклад в термическое сопротивление будут вносить: процессы теплоотдачи с горячей и холодной сторон, сопротивления теплопередачи слоёв металла, керамических пластин, термопасты и т.д. Составляющие термического сопротивления уникальны для каждой модели ТЭГ. Параметры $R_{h}$ и $R_{c}$, введённые в математическую модель, включают все компоненты термического сопротивления конструкции, что позволяет избежать ограничения применимости математической модели одной конкретной конструктивной схемой ТЭГ. Предполагается, что конструктивные термические сопротивления определяются на предшествующем этапе проектировании генератора и приводятся к площади поперечного сечения термоэлектрического слоя.

В проведённых расчётах было принято, что

${ }^{5,6}$ Appendix 1 
суммарное тепловое сопротивление конструкции ТЭГ $\left(R_{h}+R_{c}\right)$ равно сопротивлению полупроводникового слоя, а значения $R_{h}$ и $R_{c}$ равны между собой. При принятом соотношении сопротивлений изменение всех параметров на нагрузочной характеристике имеет существенную величину [29].

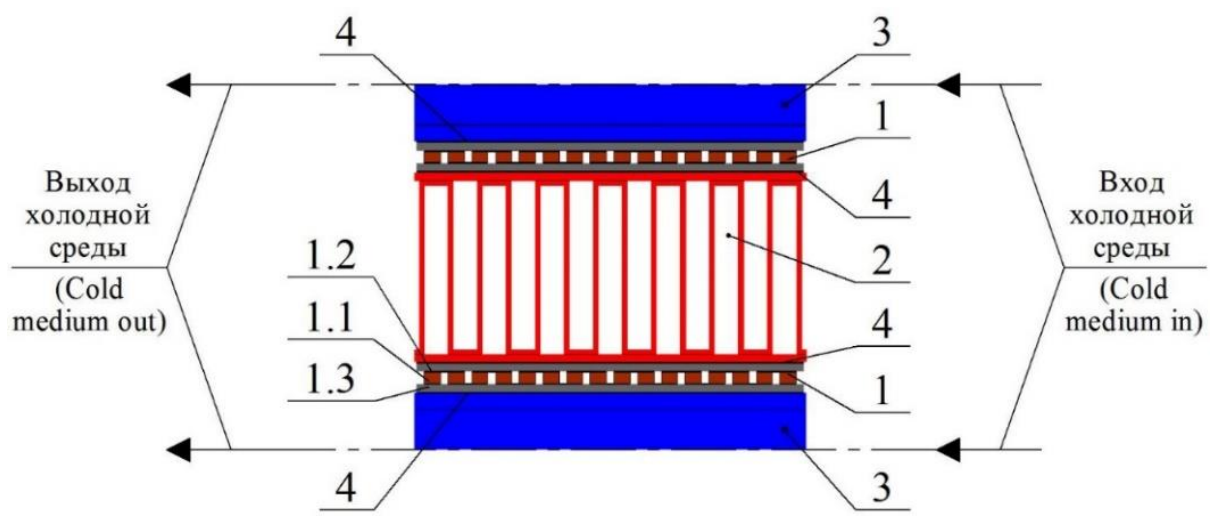

1 - ТЭМ, 1.1 - термоэлектрический слой, 1.2 - коммутационная шина, 1.3 - керамическая пластина, 2 охладитель горячей среды, 3 - нагреватель холодной среды, 4 - термопаста.

Рис. 7. Элементы конструкции ТЭГ. ${ }^{7}$

В табл. 1 приведены дополнительные исходные данные, использовавшиеся в расчётах, а также представлены значения средней температуры полупроводникового слоя в режиме холостого хода и отклонения этой температуры от оптимальной.

На рис. 8 показана полная нагрузочная характеристика для $\mathrm{Bi}_{2} \mathrm{Te}_{3}$ по параметрам математической модели. Она была построена с учётом переменности термоэлектрических свойств. По характеристике видно, как с уменьшением нагрузочного коэффициента и увеличением тока нагрузки происходит сокращение температурного перепада между спаями ТЭГ и возрастание теплового потока на горячих спаях, который начинает существенно отличаться от теплового потока теплопроводности, о чём говорит рост коэффициента термопроводности. Данные процессы приводят к изменению производительности и эффективности работы ТЭГ, которые достигают экстремумов при некоторых значениях $\bar{I}$ и $m$. Таким образом на левой части характеристики показаны макропараметры ТЭГ, а на правой - микропараметры, определяющие физические процессы.

Сопоставляя левые части характеристик, полученных при постоянных и переменных термоэлектрических свойствах, возможно, при переменном соотношении электрических сопротивлений сети и генератора, оценить степень влияния переменности свойств определённого термоэлектрического материала на макропараметры работы генератора. Для материалов из табл. 1 сопоставление характеристик с постоянными и переменными свойствами представлено на рис. 9 - рис. 11.

Таблица $1^{8}$.

Supplementary initial data for calculation of load characteristics ${ }^{9}$.

\begin{tabular}{|c|c|c|c|c|}
\hline $\begin{array}{c}\text { Материал } \\
\text { Material }\end{array}$ & $\begin{array}{c}\text { Тепловые } \\
\text { сопротивления } \\
\text { конструкции, } \\
R_{h}=R_{c} \\
\left(\mathrm{MM}^{2} \cdot \mathrm{K} / \mathrm{BT}\right) \\
\text { Hot and cold } \\
\text { side thermal } \\
\text { resistance } \\
\left(\mathrm{mm}^{2} \cdot \mathrm{K} / \mathrm{W}\right)\end{array}$ & $\begin{array}{c}\text { Температура } \\
\text { горячей среды } \\
\text { Hot medium } \\
\text { temperature } \\
\left({ }^{\circ} \mathrm{C}\right)\end{array}$ & $\begin{array}{c}\text { Средняя } \\
\text { температура } \\
\text { Mean temperature } \\
\left({ }^{\circ} \mathrm{C}\right)\end{array}$ & $\begin{array}{c}\text { Отклонение } \\
\text { средней } \\
\text { температуры от } \\
\text { оптимальной } \\
\text { Deviation of the } \\
\text { mean temperature } \\
\text { from the optimum } \\
\left({ }^{\circ} \mathrm{C}\right)\end{array}$ \\
\hline $\mathrm{Bi}_{2} \mathrm{Te}_{3}$ & 939 & 250 & 140 & 90 \\
\hline $\begin{array}{l}\mathrm{Bi}_{2} \mathrm{Te}_{3}-\mathrm{Sb}_{2} \mathrm{Te}_{3} / \\
\mathrm{Bi}_{2} \mathrm{Te}_{3}-\mathrm{Bi}_{2} \mathrm{Se}_{3} \\
\end{array}$ & 1387 & 600 & 315 & 165 \\
\hline $\mathrm{PbTe}$ & 575 & 650 & 340 & 90 \\
\hline
\end{tabular}



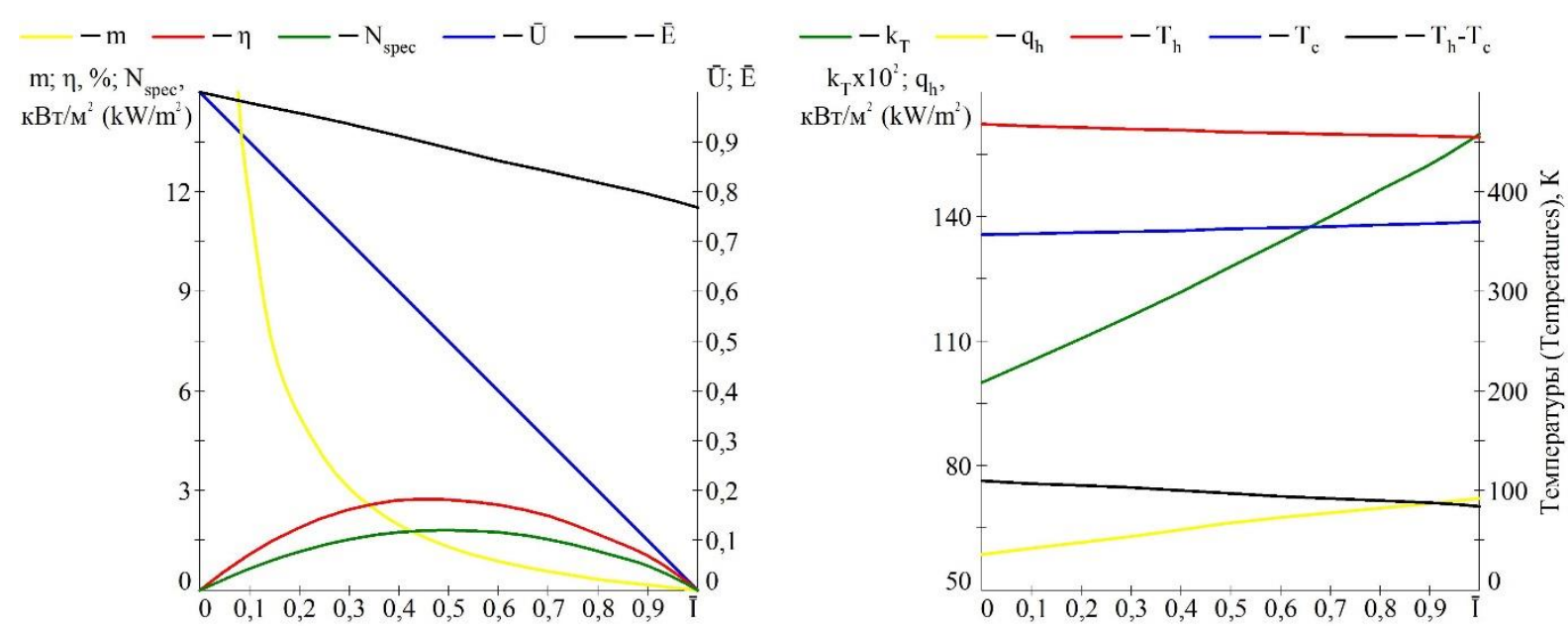

Рис. 8. Нагрузочная характеристика для $\mathrm{Bi}_{2} \mathrm{Te}_{3 .}{ }^{10}$

Из рис. 6 видно, что для $\mathrm{Bi}_{2} \mathrm{Te}_{3}$ средняя температура принята существенно меньше предельной, поскольку необходимо учитывать, что вблизи горячего спая температура будет существенно выше и может способствовать разрушению материала.

Для материала из твёрдых растворов средняя температура наоборот завышена, что существенно снижало бы ресурс реального ТЭГ. Высокая средняя температура необходима, чтобы продемонстрировать влияние температурных зависимостей на нагрузочную характеристику, которое для этого материала, как будет показано далее, мало. Для РbТе приближение средней температуры к предельной по рис. 6 не критично, так как в соответствии с [28] свойства РbTе приведены вплоть до 900 К, а на рис. 6 показана лишь часть диапазона,

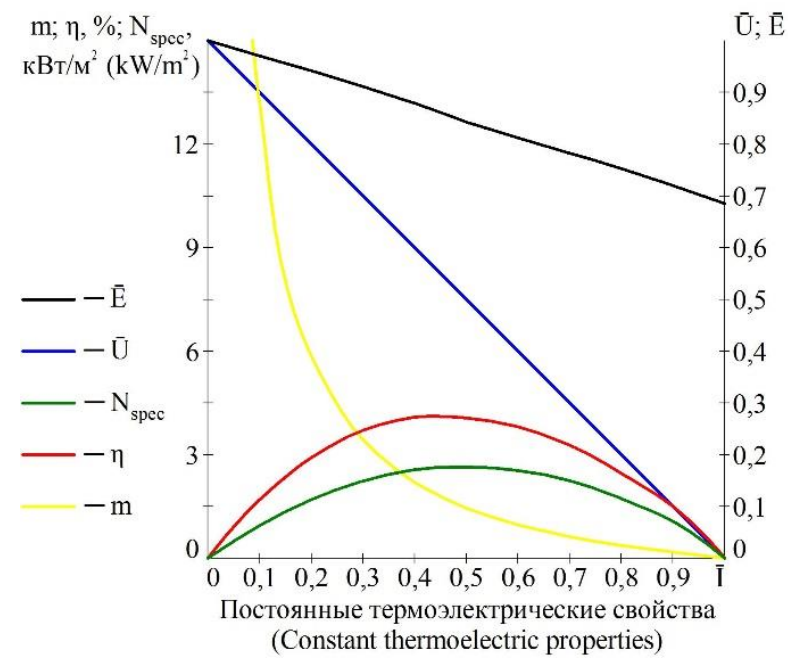

востребованная для судовых теплоутилизационных систем.

У ТЭГ с термоэлектрическим слоем из $\mathrm{Bi}_{2} \mathrm{Te}_{3}$ при возрастании $\bar{I}$ от 0 до 1 температура горячих спаев снижается примерно на 15 К, температура холодных спаев - возрастает на туже величину; для других материалов изменения составляют порядка 40 К. С изменениями температуры спаев меняется и средняя температура полупроводникового слоя, а значит и термоэлектрические свойства.

Во всех случаях результаты, полученные с учётом переменности термоэлектрических свойств, были хуже, т.к. отклонение средней температуры от оптимальной означает снижение термоэлектрической добротности, а значит и $\eta$ и $N_{\text {spec }}$ в соответствии с формулами (5), (7).

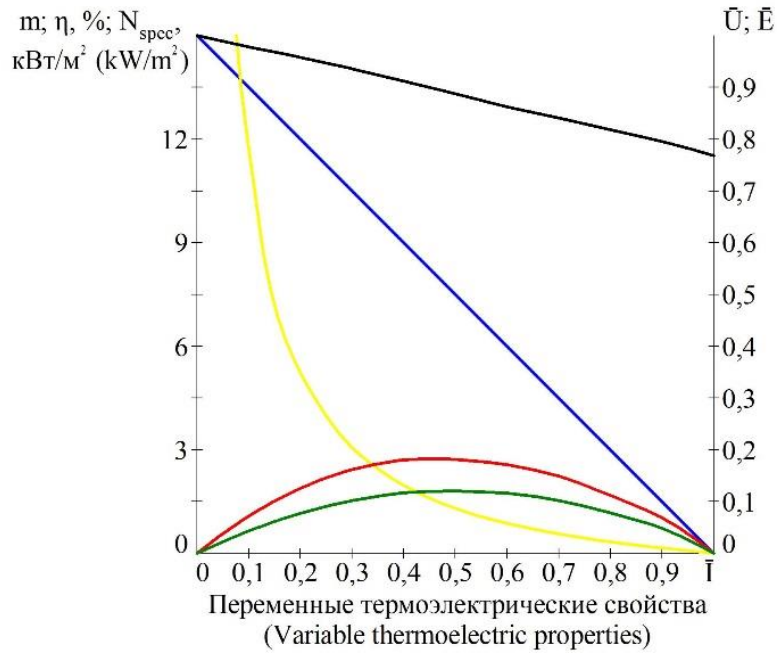

Рис. 9. Сопоставление характеристик для Вi2 $2 \mathrm{Te}_{3}{ }^{11}$ 

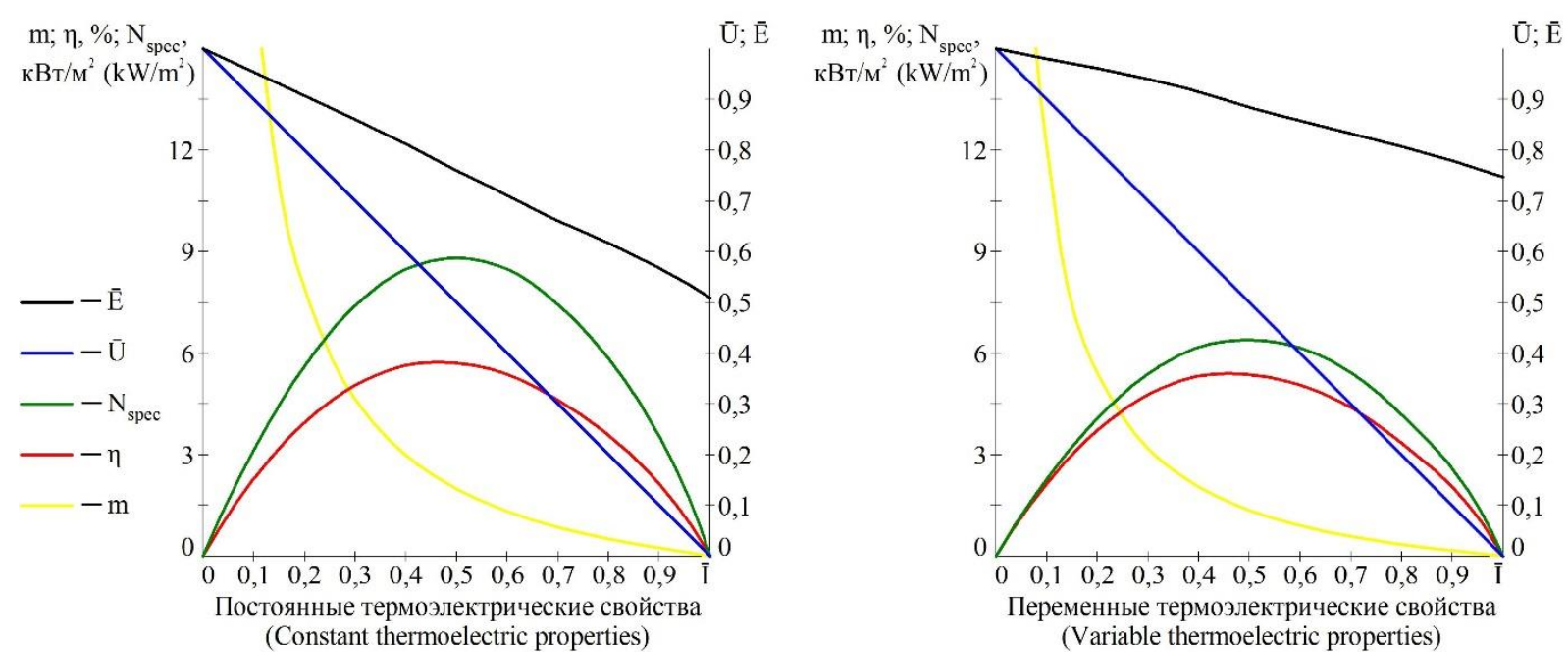

Рис. 10. Сопоставление характеристик для твёрдых растворов. ${ }^{12}$
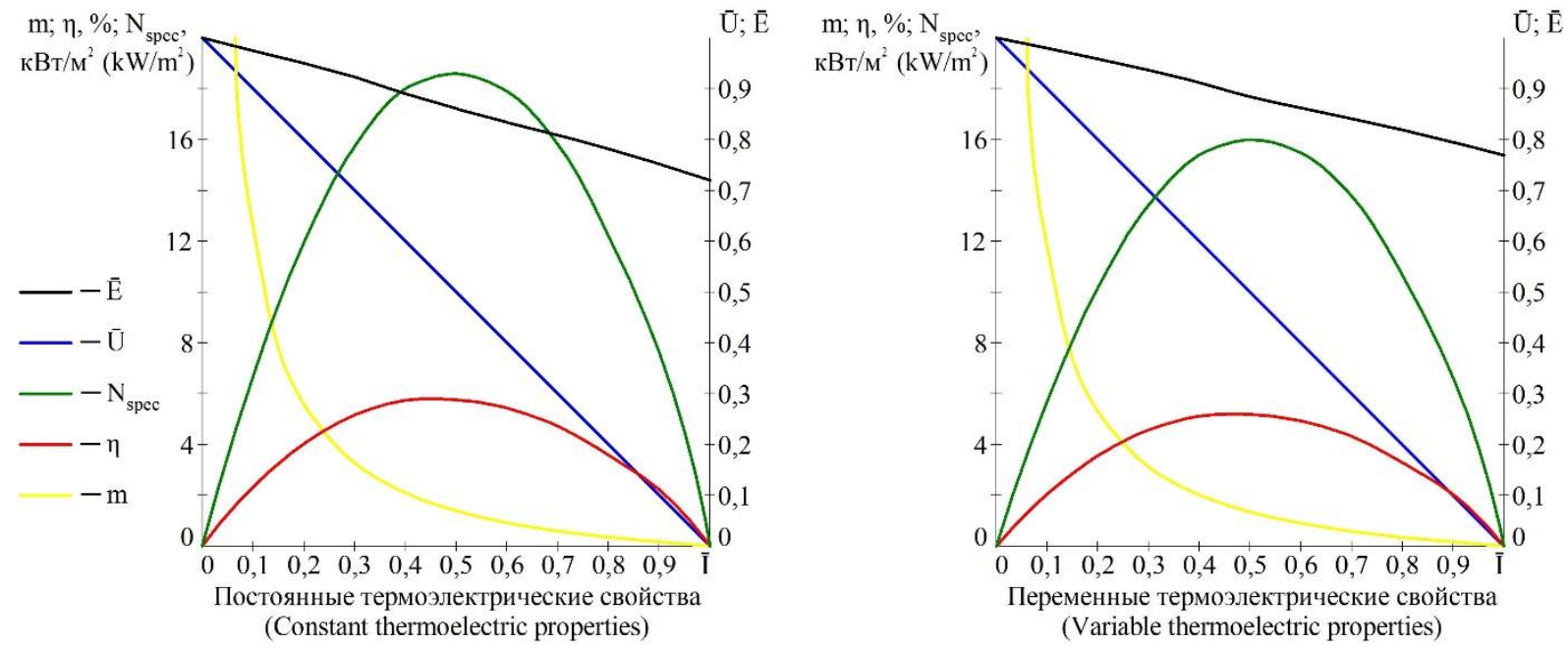

Рис. 11. Сопоставление характеристик для РbTe. ${ }^{13}$

Если при существенном перепаде температуры на ТЭГ средняя температура полупроводникового слоя будет близка к оптимальной, то генератор будет работать достаточно эффективно. В режиме максимального КПД наибольшее его снижение происходило у $\mathrm{Bi}_{2} \mathrm{Te}_{3}:$ с 4,09\% до 2,71\%. Для материала из твёрдых растворов изменение КПД из-за учёта термоэлектрических свойств минимально, и некоторую разницу удалось получить лишь при предельном значении средней температуры: КПД уменьшился с 5,71\% до 5,37\%. Для $\mathrm{PbTe}$ изменение КПД составило 10\%.

\section{Заключение}

Была разработана математическая модель расчёта нагрузочной характеристики термоэлектрического генератора, учитывающая влияние температурных зависимостей термоэлектрических свойств на эксплуатационные параметры. Она может быть применена для анализа эффективности использования термоэлектрических материалов в конструкции существующих генераторов, для обеспечения оптимального выбора материалов при новом конструировании и для прогнозирования изменения параметров ТЭГ при внедрении перспективных материалов.

Из результатов расчётов, приведённых в работе, следует что принимаемые конструктивные решения могут существенно влиять на эффективность использования термоэлектрического материала. Например, среди рассмотренных материалов наилучшими термоэлектрическими свойствами, при оптимальной средней температуре, обладает полупроводниковый слой из твёрдых растворов, однако, при принятых расчётных условиях, ТЭГ с его применением показал эффективность на уровне генератора на базе теллурида свинца.

Из приведённых нагрузочных характеристик следует, что КПД и удельная мощность 
ТЭГ существенно изменяются с изменением тока нагрузки. При работе генератора необходимо поддерживать требуемое соотношение между электрическими сопротивлениями нагрузки и самого ТЭГ, что может быть обеспечено, например, применением преобразователей постоянного тока $[31,32]$.

\section{APPENDIX 1 (ПРИЛОЖЕНИЕ 1)}

${ }^{1}$ Fig. 1. The scheme of the algorithm elaborated for the calculation of a load diagram.

${ }^{2}$ Fig. 2. The schematic diagram of a thermoelectric generator.

${ }^{3} \mathrm{Fig}$. 3. The thermoelectric properties of $\mathrm{Bi}_{2} \mathrm{Te}_{3}$ layer.

${ }^{4} \mathbf{F i g}$. 4. The thermoelectric properties of $\mathrm{Bi}_{2} \mathrm{Te}_{3}-\mathrm{Sb}_{2} \mathrm{Te}_{3}$ and $\mathrm{Bi}_{2} \mathrm{Te}_{3}-\mathrm{Bi}_{2} \mathrm{Se}_{3}$ layers.

${ }^{5}$ Fig. 5. The thermoelectric properties of $\mathrm{PbTe}$ layer.

${ }^{6}$ Fig. 6. The graphs of the dimensionless figure of merit for the applied materials.

${ }^{7}$ Fig. 7. Construction elements of a thermoelectric generator. ( 1 - thermoelectric module, 1.1 - thermoelectric layer, 1.2 - electrical conductor, 1.3 - ceramic plate, 2 - hot sink, 3 - cold sink, 4 - thermal grease).

${ }^{8,9}$ Table 1. Auxiliary parameters for the calculation of the load diagrams.

${ }^{10}$ Fig. 8. The load diagram for $\mathrm{Bi}_{2} \mathrm{Te}_{3}$.

${ }^{11}$ Fig. 9. The comparison of the diagrams with constant and variable thermoelectric properties for $\mathrm{Bi}_{2} \mathrm{Te}_{3}$.

${ }^{12}$ Fig. 10. The comparison of the diagrams with constant and variable thermoelectric properties for the solid solutions.

${ }^{13}$ Fig. 11. The comparison of the diagrams with constant and variable thermoelectric properties for $\mathrm{PbTe}$.

\section{Литература (References)}

[1] Ioffe A.F. Poluprovodnikovye Termoelementy [Semiconductor Thermoelements]. Moscow Leningrad, Academy of Science of the USSR, 1960. $189 \mathrm{p}$.

[2] Hendricks T., Choate W.T. Engineering Scoping Study of Thermoelectric Generator Systems for Industrial Waste Heat Recovery. U.S. Department of Energy. 2006. 74 p.

[3] Liu C., Chen P., Li K. A 500 W Low-Temperature Thermoelectric Generator: Design and Experimental Study. International Journal of Hydrogen Energy, 2014, vol. 39, no. 28, pp. 15497-15505. doi: 10.1016/j.ijhydene.2014.07.163

[4] Chen J., Zuo L., Wu Y., Klein J. Modeling, Experiments and Optimization of an on-Pipe Thermoelectric Generator. Energy Conversion and Management, 2016, vol. 122, pp. 298-309. doi: 10.1016/j.enconman.2016.05.087

[5] Risseh A.E., Nee H.-P., Erlandsson O., Brinkfeldt K., Contet A., Frobenius F., Gaiser G., Saramat A., Skare T., Nee S., Dellrud J. Design of a Thermoelectric Generator for Waste Heat Recovery Application on a Drivable Heavy Duty Vehicle.
SAE International Journal of Commercial Vehicles, 2017, vol. 10, no. 1, pp. 26-44. doi: 10.4271/2017-01-9178

[6] Chen J., Lin B., Wang H., Lin G. Optimal Design of a Multi-Couple Thermoelectric Generator. Semicond. Sci. Technol., 2000, vol. 15, no. 2, pp. 184-188. doi: 10.1088/0268-1242/15/2/318

[7] Al-Habahbeh O.M., Mohammad A., Al-Khalidi A., Khanfer M., Obeid M. Design Optimization of a Large-Scale Thermoelectric Generator. Journal of King Saud University, 2018, vol. 30, no. 2, pp. 177-182. doi: 10.1016/j.jksues.2016.01.007

[8] Tzeng S.-C., Jeng T.-M., Lin Y.-L. Parametric Study of Heat-Transfer Design on the Thermoelectric Generator System. International Communications in Heat and Mass Transfer, 2014, vol. 52, pp. 97-105. doi: 10.1016/j.icheatmasstransfer.2014.01.021

[9] Ramade P., Patil P., Shelar M., Chaudhary S., Yadav S., Trimbake S. Automobile Exhaust Thermo-Electric Generator Design \& Performance Analysis. International Journal of Emerging Technology and Advanced Engineering, 2014, vol. 4, no. 5, pp. 682-691.

[10] Ouyang Z., Li D. Modelling of Segmented Highperformance Thermoelectric Generators with Effects of Thermal Radiation, Electrical and Thermal Contact Resistances. Sci. Rep., 2016, no. 6: 24123. doi: 10.1038/srep24123

[11]Crane D.T., Kossakovski D., Bell L.E. Modeling the Building Blocks of a 10\% Efficient Segmented Thermoelectric Power Generator. Journal of ELECTRONIC MATERIALS, 2009, vol. 38, no. 7, pp. 1382-1386. doi: 10.1007/s11664-009-0673-y

[12] Ge Y., Liu Z., Sun H., Liu W. Optimal Design of a Segmented Thermoelectric Generator Based on Three-Dimensional Numerical Simulation and Multi-Objective Genetic Algorithm. Energy, 2018, vol. 147,pp. 1060-1069. doi: 10.1016/j.energy.2018.01.099

[13]Zhang G., Fan L., Niu Z., Jiao K., Diao H., Du Q., Shu G. A Comprehensive Design Method for Segmented Thermoelectric Generator. Energy Conversion and Management, 2015, vol. 106, pp. 510519. doi: 10.1016/j.enconman.2015.09.068

[14] Kim H.S., Kikuchi K., Itoh T., Iida T., Taya M. Design of Segmented Thermoelectric Generator Based on Cost-Effective and Light-Weight Thermoelectric Alloys. Materials Science and Engineering B, 2014, vol. 185 , no. 1, pp. 45-52. doi: 10.1016/j.mseb.2014.02.005

[15] Chen L., Li J., Sun F., Wu C. Performance Optimization of a Two-Stage Semiconductor Thermoelectric-Generator. Applied Energy, 2005, vol. 82, no. 4, pp. 300-312. doi: 10.1016/j.apenergy.2004.12.003

[16] Ma Q., Fang H., Zhang M. Theoretical Analysis and Design Optimization of Thermoelectric Generator. Applied Thermal Engineering, 2017, 
vol. 127, pp. 758-764. doi: 10.1016/j.applthermaleng.2017.08.056

[17] Meng J.-H., Wang X.-D., Chen W.-H. Performance Investigation and Design Optimization of a Thermoelectric Generator Applied in Automobile Exhaust Waste Heat Recovery. Energy Conversion and Management, 2016, vol. 120, pp. 71-80. doi: 10.1016/j.enconman.2016.04.080

[18]He W., Wang S., Zhang X., Li Y., Lu C. Optimization Design Method of Thermoelectric Generator Based on Exhaust Gas Parameters for Recovery of Engine Waste Heat. Energy, 2015, vol. 91, pp. 1-9. doi: 10.1016/j.energy.2015.08.022

[19] Niu Z., Diao H., Yu S., Jiao K., Du Q., Shu G. Investigation and Design Optimization of ExhaustBased Thermoelectric Generator System for Internal Combustion Engine. Energy Conversion and Management, 2014, vol. 85, pp. 85-101. doi: 10.1016/j.enconman.2014.05.061

[20]Jia X., Gao Y. Optimal Design of a Novel Thermoelectric Generator with Linear-Shaped Structure under Different Operating Temperature Conditions. Applied Thermal Engineering, 2015, vol. 78, pp. 533-542. doi: 10.1016/j.applthermaleng.2014.12.011

[21]Luo D., Sun Z., Wang R. Performance Investigation of a Thermoelectric Generator System Applied in Automobile Exhaust Waste Heat Recovery. Energy, 2022, vol. 238, unpublished. doi: 10.1016/j.energy.2021.121816

[22] Ahiska R., Mamur H. Design and Implementation of a New Portable Thermoelectric Generator for Low Geothermal Temperatures. IET Renewable Power Generation, 2013, vol. 7, no. 6, pp. 700706. doi: 10.1049/iet-rpg.2012.0320

[23] Narjis A., Liang C.-T., El Aakib H., Tchenka A, Outzourhit A. Design Optimization for Maximized Thermoelectric Generator Performance. Journal of Electronic Materials, 2020, vol. 49, no. 1, pp. 306-310. doi: 10.1007/s11664-019-07748-9

[24] Qing S., Rezaniakolaei A., Rosendahl L.A., Gou X. An Analytical Model for Performance Optimi-

\section{Сведения об авторах.}

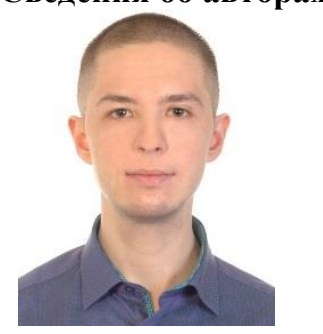

\section{Голубев Роман Олего- вич, аспирант, СПбГМТУ. Область научных инте- ресов: энергоэффек- тивность, теплообмен- ные аппараты, судовые энергетические уста- новки. \\ E-mail: $\underline{\text { swit.roma@mail.ru }}$}

zation of Thermoelectric Generator with Temperature Dependent Materials. IEEE Access, 2018, vol.6,pp.60852-60861.doi: 10.1109/ACCESS.2018.2874947

[25] Meng F., Chen L., Sun F. Effects of Temperature Dependence of Thermoelectric Properties on the Power and Efficiency of a Multielement Thermoelectric Generator. International Journal of Energy and Environment, 2012, vol. 3, no. 1, pp. 137-150.

[26] Rowe D.M. Thermoelectrics Handbook: Macro to Nano. Boca Raton, CRC Press. 2006. 954 p.

[27] Ohotin A.S., Efremov A.A., Ohotin V.S., Pushkarskij A.S. Termoelektricheskie Generatory [Thermoelectric Generators]. Moscow, Atomizdat Publ., 1971. 288 p.

[28] Cadoff I.B., Miller E. Thermoelectric Materials and Devices. New York, Reinhold, 1960. 344 p. (Russ. ed.: Karchevskij A.I. Termoelektricheskie Materialy i Ppreobrazovateli. Moscow, Mir Publ., 1964. 352 p.).

[29] Kowalsky R.V. Inzhenernye Metody Raschota Termoelektricheskih Generatorov [Engineering Calculation Methods for Thermoelectric Generators]. Moscow, Nauka Publ., 1990. 189 p.

[30] Vihorev G.A., Semenyuk V.A. Ratsional'noe Razmeshchenie Termoelementov v Ohlazhdayushchej Poluprovodnikovoj Termobataree [Rational Positioning of Thermoelements in a Semiconductor Thermoelectric Cooler]. Holodil'naya Tekhnika i Tekhnologiya - Refrigeration Engineering and Technology, 1966, no. 2, pp. 7-15. (In Russian).

[31] Eakburanawat J., Boonyaroonate I. Development of a Thermoelectric Battery-charger with Microcontroller-Based Maximum Power Point Tracking Technique. Applied Energy, 2006, vol. 83, no. 7, pp. 687-704. doi: 10.1016/j.apenergy.2005.06.004

[32] Yu C., Chau K.T. Thermoelectric Automotive Waste Heat Energy Recovery Using Maximum Power Point Tracking. Energy Conversion and Management, 2009, vol. 50, no. 6, pp. 1506-1512. doi: 10.1016/j.enconman.2009.02.015

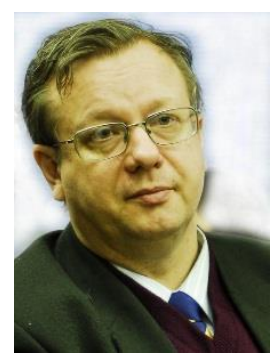

Столяров Сергей Павлович,

кандидат технических наук, доцент, заведующий кафедрой судовых ДВС и дизельных установок СПбГМТУ.

Область научных интересов: процессы теплообмена и газодинамики, тепловые трубы, двигатели внутреннего сгорания и с внешним подводом теплоты. E-mail: stsp56@yandex.ru 